\title{
Changements climatiques et ressources en eau Bassin versant de l'Ouergha (Maroc)
}

\author{
Climate change and water resources \\ Ouergha watershed (Morocco)
}

\author{
S. Senoussi ${ }^{(1)}$, A. Agoumi (2), M. Yacoubi (3), A. Fakhraddine ${ }^{(4)}$, \\ E.H. Sayouty ${ }^{(5)}, A$. Mokssit $^{(6)}$, N. Chikri( ${ }^{(7)}$
}

(1) Doctorante en Thèse Nationale, Université Hassan II - Faculté des Sciences Aïn Chok Département de Physique - BP: 5366 Maârif - Km 8 Route d'El Jadida - Casablanca - Maroc / Etudiante - chercheur au Laboratoire Public d'Essais et d'Etudes - Centre de Réalisation de la Recherche dans le domaine de l'Eau et l'Environnement (CRR / EE) - Km 7 Route d'El Jadida Oasis - Casablanca - Maroc.

(2) Professeur à l'Ecole Hassania des Travaux Publics - BP : 8108 Oasis- Casablanca - Maroc / Chercheur associé au Laboratoire Public d'Essais et d'Etudes (CRR / EE).

(3) Ingénieur hydrologue au Laboratoire Public d'Essais et d'Etudes - Centre de Réalisation de la Recherche dans le domaine de l'Eau et l'Environnement (CRR / EE).

(4) Chef du Centre de Réalisation de la Recherche dans le domaine de l'Eau et l'Environnement (CRR / EE).

(5) Professeur à l'Université Hassan II - Faculté des Sciences Aïn Chok - Département de Physique - B P: 5366 Maârif - Km 8 Route d'El Jadida - Casablanca - Maroc.

(6) Chef de service à la Direction de la Météorologie Nationale - Casablanca - Maroc.

(7) Ingénieur à la Direction Générale de I'Hydraulique - Rue Hassan Benchekroune, Agdal Rabat - Maroc.

Résumé. - Les changements climatiques, prévus avec l'augmentation des gaz à effet de serre, pourraient avoir un impact certain sur les ressources en eau, en particulier dans les régions arides à semi-arides, comme celle du Maghreb.

Tenant compte des connaissances actuelles concernant le réchauffement probable de la région, et en vue d'évaluer l'impact possible sur la ressource en eau de tels événements, la recherche dont les résultats sont présentés ici, a consisté à :

- Simuler à l'aide d'un modèle, le comportement hydrique d'un bassin versant pilote au Maroc (bassin de l'Ouergha)

- Utiliser ce modèle pour estimer le déficit hydrique probable en cas de changement climatique dans ce bassin

Les premiers résultats indiquent une diminution des ruissellements annuels à l'exutoire de ce bassin atteignant les $8 \%$ d'ici à 2050: déficit important dans un pays où la ressource est particulièrement rare.

Mots clés. - Bassin, climat, eau, hydrologie, réchauffement. 


\begin{abstract}
When climatic changes occur, they can have a marked impact on water resources, particularly in arid to semi-arid regions like the Maghreb.

The study presented here, which draws on present knowledge relating to the probable warming of the region, aimed at assessing the possible impact of such events on water resources and consisted in:

- modeling the hydric behaviour of one watershed in Morocco (Ouergha basin)

- using this model to estimate the probable hydric deficit in the event of climatic change in the basin.

The initial results indicate an annual decrease in streaming at the outlet from the basin. By 2050 , this decrease could reach $8 \%$, which is considerable in an area where water resources are particularly limited.
\end{abstract}

Key words. - Watershed, climate, water resources, hydrology, warming.

\section{INTRODUCTION}

L'eau est un élément rare et un facteur essentiel qui conditionne fortement l'avenir de la région du Maghreb, et ce, indépendamment de tout changement climatique : au centre du Maghreb, le Maroc est un pays en stress hydrique qui devrait connaître, au-delà de 2025, une situation de pénurie d'eau [Agoumi, 1998], [Perennes, 1993].

Le climat de la région a connu un réchauffement significatif depuis les années 1970. Ce réchauffement s'est accompagné particulièrement au Maroc d'une diminution nette des précipitations (jusqu'à 1995). Pendant ces décennies, le bilan en eau de la région était très déficitaire, et la problématique de l'eau s'est posée avec acuité [Agoumi, 1995].

Les prévisions des modèles de circulation générale (MCG), concernant l'évolution possible du climat planétaire en cas de changement climatique, prévoient pour cette région un réchauffement pouvant atteindre les $4^{\circ} \mathrm{C}$ entre 2000 et 2100 . Pour l'avenir des précipitations, les résultats des modèles sont moins précis, ils indiquent cependant une légère tendance à la baisse pour la partie sud de la Méditerranée [Karas, 1997].

Si ces changements climatiques se concrétisaient, le bilan en eau de la région deviendrait encore plus fragile et l'enjeu eau plus critique. Les actions à mener par ces pays pour parer aux conséquences possibles des changements climatiques, en particulier sur les ressources en eau, nécessitent une connaissance quantifiée de ces impacts.

C'est dans ce cadre que s'inscrit ce projet de recherche initié au Maroc en 1996 par la Direction Générale de l'Hydraulique, la Direction de la Météorologie Nationale et le Laboratoire Public d'Essais et d'Etudes. L'objectif du projet était de quantifier l'impact possible des changements climatiques sur les eaux mobilisées par le Maroc au niveau des réservoirs de barrage. Le site pilote choisi pour cette recherche est le bassin versant de l'Ouergha, alimentant le plus grand 
barrage du royaume: Al Wahda (figure 2).

Un modèle simulant les ruissellements à l'exutoire de ce bassin, tenant compte des précipitations et des températures de l'air in situ a été développé, calibré et validé. Dans une seconde phase, cet outil a été utilisé pour prévoir le devenir des ruissellements en cas de changements climatiques.

\section{MODÈLE DÉVELOPPÉ}

Le premier objectif de cette recherche a été d'arriver à une simulation correcte des débits journaliers en un point d'une rivière (exutoire du bassin versant) à partir de la connaissance de la pluie journalière moyenne reçue par le bassin versant, et de l'évaporation potentielle, en utilisant une représentation simple des processus pluie-débit, faisant intervenir un nombre limité de paramètres à caler [Nascimento, 1995].

Le modèle utilisé est un modèle conceptuel, à 9 paramètres et à pas de temps journalier [H.O.M, 1989]. II est basé sur une représentation simple des processus qui gouvernent l'écoulement de l'eau dans les rivières.

Le lien remarquable entre l'intensité des pluies et l'importance de l'écoulement qu'elles produisent est exprimé mathématiquement dans ce modèle, par une série d'équations. Celui-ci utilise le concept de "réservoirs" pour prendre en considération

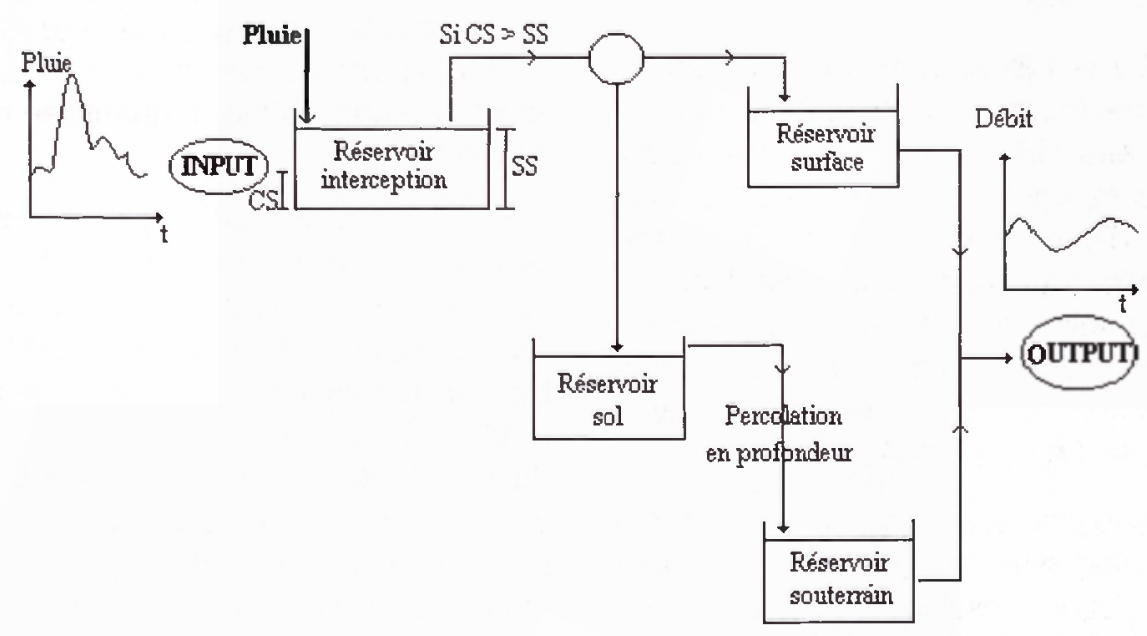

Figure 1. - Schéma conceptuel du modèle.

Fig. 1.Conceptual diagram of model. 
le passage de l'eau à travers le bassin versant. Le schéma conceptuel du modèle est présenté sur la figure 1.

Les réservoirs utilisés sont au nombre de 4 :

- Le réservoir - interception;

- Le réservoir - surface;

- Le réservoir - sol;

- Le réservoir - souterrain.

\section{Réservoir - interception}

Ce réservoir répartie la pluie nette sur tous les réservoirs. II a pour entrée les précipitations et pour sortie le prélèvement occasionné par l'èvaporation, il permet de répartir la pluie nette entre lui-même et les autres réservoirs.

\section{Réservoir - surface}

Ce réservoir reçoit une partie du débit sortant du réservoir-interception et le transforme en ruissellement direct sur le bassin versant.

\section{Réservoir - sol}

Ce réservoir représente le processus d'emmagasinement sous forme d'humidité du sol. II commande le phénomène de saturation du sol et permet la propagation du débit jusqu'au dernier réservoir. Il est alimenté par infiltration, à partir du réservoir interception, qui réduit ainsi le déficit d'humidité du sol.

Le présent réservoir est réduit par transpiration. Ce phénomène biologique (transpiration), par lequel les plantes absorbent une quantité d'eau du sol qui est restituée partiellement sous forme de vapeur par la surface des feuilles, n'aura lieu que si l'évaporation dépasse le volume d'eau contenu dans le réservoir-interception. En effet, l'extraction de l'eau par évapotranspiration, selon le modèle, se fait d'abord par transformation du liquide sur la surface, en vapeur (évaporation), puis, si le phénomène évaporatoire persiste, il va puiser l'eau jusque dans les plantes (transpiration).

\section{Réservoir - souterrain}

Ce réservoir reçoit le débit de sortie du réservoir - sol et le transforme en débit de base.

Le passage d'eau du réservoir sol vers le réservoir souterrain se fait par percolation en profondeur, à condition que le déficit d'humidité soit négatif.

\section{PRÉSENTATION DU BASSIN VERSANT DE L'OUERGHA}

Le site d'étude sur lequel a porté notre choix est le bassin versant de l'Ouergha (figure 2). Les raisons de ce choix sont les suivantes :

- La contribution de ses nappes phréatiques y est faible, il est donc relativement simple à modéliser;

- Il est suffisamment hétérogène en surface pour être représentatif d'une grande variété de situations; 


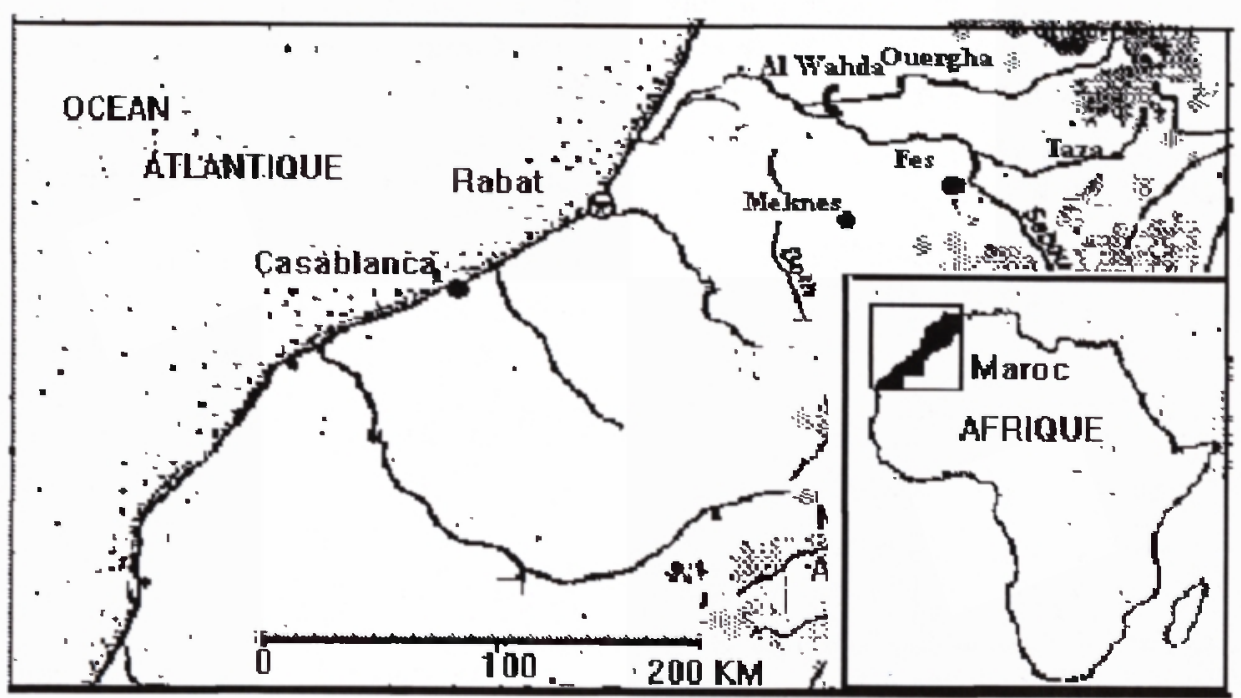

Figure 2. - Présentation du site d'étude.

Fig. 2. - Presentation of study site.

- Le bassin alimente le plus grand barrage du royaume, appréhender son comportement vis à vis des changements climatiques, c'est déjà connaître le devenir du $1 / 3$ des eaux mobilisées du pays.

Le bassin versant de l'Ouergha est situé entre les monts subrifains au Nord et les monts prérifains au sud. Ce bassin, qui occupe une superficie de $6190 \mathrm{~km}^{2}$, se trouve en amont de la station hydrométrique M'jara, et draine la partie sud de la chaîne du Rif (figure 3).

Le bassin peut être divisé en 3 zones topographiques distinctes: les hautes montagnes (altitude $>1200 \mathrm{~m}$ ), les montagnes d'altitude moyenne (400 $\mathrm{m}<$ altitude $<1200 \mathrm{~m}$ ) et les collines et plaines des vallées (altitude $<400 \mathrm{~m}$ ).

Son climat est du type méditerranéen avec une pluviométrie qui peut aller jusqu'à $1800 \mathrm{~mm} / \mathrm{an}$ dans les régions montagneuses; il s'agit ainsi des zones les plus humides du royaume.

La zone du bassin de l'Ouergha, en amont de la station hydrométrique M'jara, est principalement composée de terres argileuses ainsi que de formations de schistes et de marnes du mésozoïque.

La prédominance des terres argileuses et la présence des couches désagrégées de marnes et de schistes augmente l'imperméabilité du bassin versant, et en fait un sol à ca- 


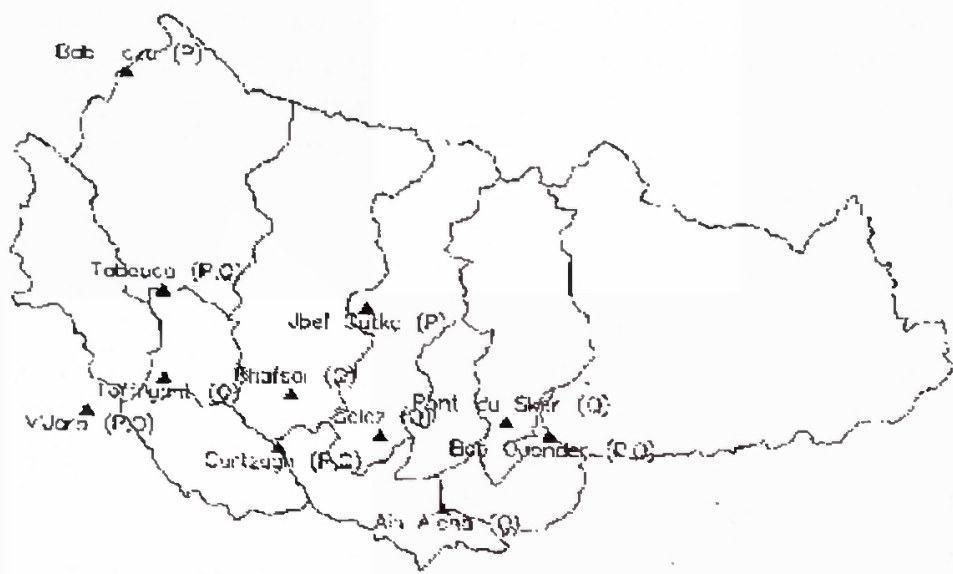

Figure 3. - Bassin versant de l'Ouergha.

Fig. 3. - Ouergha watershed.

pacité de rétention non négligeable, ce qui laisse prévoir la présence d'une faible nappe phréatique.

L'étude de ce bassin est d'autant plus importante qu'on retrouve à son exutoire le barrage Al Wahda, la retenue la plus importante du royaume $\left(2^{\mathrm{e}}\right.$ au niveau de l'Afrique) de point de vue capacité de stockage $(3,7$ milliards de $\mathrm{m}^{3}$ ). L'apport moyen annuel est de $3300 \mathrm{Mm}^{3}$ avec un module annuel de $105 \mathrm{~m}^{3} / \mathrm{s}$ environ. Les crues du bassin qui se produisent entre octobre et avril sont parfois d'une extrême violence [DGH, 1995].

\section{DONNÉES D'ENTRÉE DU MODĖLE}

Les données nécessaires pour le fonctionnement du modèle sont la pluie journalière reçue par le bassin, le débit journalier à l'exutoire du bassin ainsi que l'évapotranspiration journalière moyenne. La reconstitution de cette base de données a nécessité un traitement de données préalable. La période d'étude utilisée est 19661995.

\subsection{Données de pluies}

Les données de précipitations nécessaires au fonctionnement du modèle sont les apports journaliers en précipitations de l'ensemble du bassin, de 1966 à 1995. Elles ont été estimées à partir des données de précipitations enregistrées au niveau de 6 stations météorologiques réparties sur le bassin versant par la méthode de Thiessen: Le bassin de l'Ouergha a été subdivisé en 11 sous - bassins, caractérisés chacun par 
une superficie, et représentés par un certain nombre de stations pluviométriques, avec un coefficient de pondération (coefficient de Thiessen) correspondant. Les stations pluviométriques utilisées sont au nombre de 6, il s'agit de Bab Ouender, Bab Taza, Jbel Outka, Tabouda et M'jara (figure 3).

Certaines de ces stations utilisées ne disposent pas de bases de données complètes. Notre premier travail a donc consisté à reconstituer ces données manquantes, en essayant tout d'abord de chercher les facteurs de corrélation entre les différentes stations.

Au terme de cette étape de reconstitution, nous avons pu disposer de séries de données complètes pour les 6 stations citées précédemment. Ces séries ont été utilisées par la suite afin de constituer les données de précipitations moyennes reçues par le bassin, et ce à l'aide de la formule suivante [DGH, 1995] :

$$
P_{\text {bassin }}(j)=\sum_{\text {station }} P_{\text {station }}^{2}(j) * \int_{\text {station }}^{\text {bassin }}
$$

$P_{\text {bassin }}$ : précipitations reçues par le bassin,

$\mathrm{P}_{\text {station }}$ : précipitations mesurées à la station,

$\int_{\text {station }}^{\text {bassin }}$ : facteur de Thiessen pour la station et le bassin concernés.

Sur la figure 4, nous avons tracé l'évolution interannuelle des précipitations moyennes reçues par le bassin de l'Ouergha sur la période 19661995 reconstituées comme décrit précédemment.

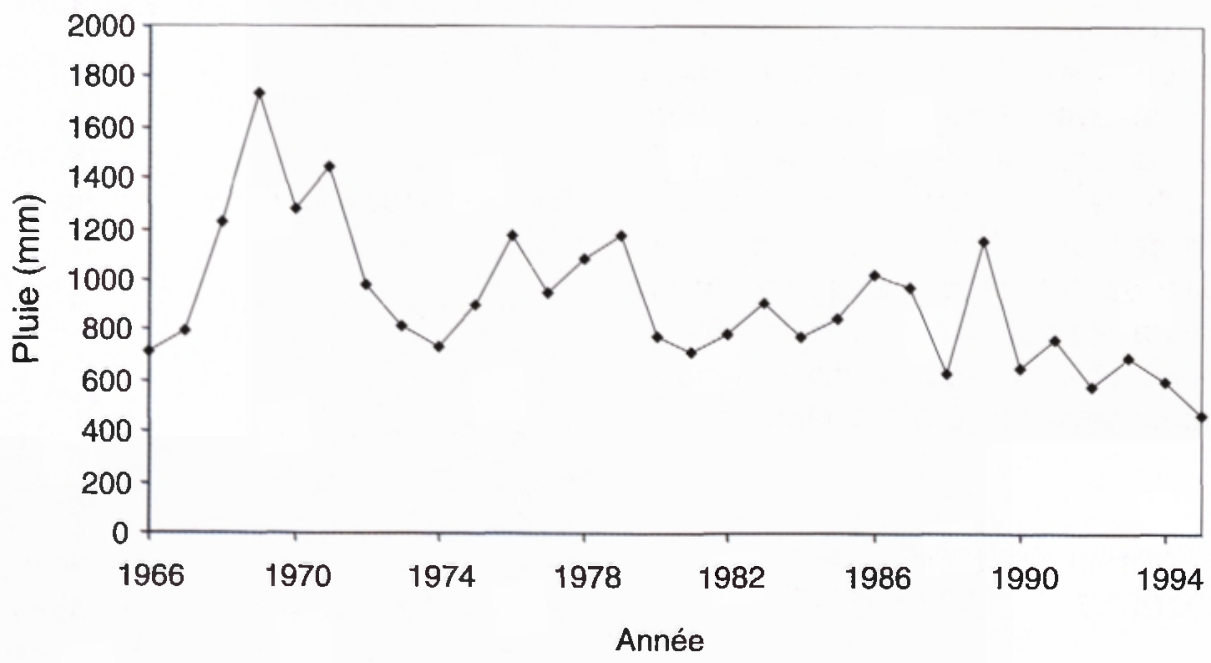

Figure 4. - Evolution interannuelle des précipitations moyennes reçues par l'Ouergha sur la période 1966-1995.

Fig. 4. - Interannual evolution of mean rainfall in Ouergha watershed (1966-1995). 
- On note une alternance de périodes sèches et humides, de périodicité variant de 5 à 10 ans.

- Les apports pluviométriques enregistrés par le bassin ont eu tendance à diminuer de façon continue. Cette diminution des apports des années 60 aux années 90 a été constaté au niveau de tout le royaume. Au niveau de l'Algérie, cette même diminution a été observée dans les zones occidentales et centrales, par contre, cette tendance n'a pas été relevée au niveau de la Tunisie et des régions orientales de l'Algérie. Les années qui ont suivi (96-97) ont été particulièrement humides, et cette tendance à la baisse semble être au moins provisoirement arrêtée.

- La période 1990-95 a connu les niveaux de précipitations les plus bas, avoisinant les $600 \mathrm{~mm}$ : 2 à 3 fois moins que les précipitations enregistrées durant les années 70 .

\subsection{Données d'évapotranspiration}

L'évapotranspiration est un facteur jouant un rôle essentiel dans les modèles de bilan hydrologique des bassins versants. Dans ces modèles, on a généralement recours à une formulation mathématique pour estimer ce facteur, connaissant l'évolution de certains paramètres caractérisant le climat du bassin.

Du fait de son rôle important dans les modèles de bilan, et en vue d'une utilisation adéquate de ce paramètre dans notre modèle, une étude spécifique portant sur l'estimation de ce facteur pour la région du Maghreb a été effectuée [Agoumi et al., 1997 ].

Plusieurs formulations trouvées dans la littérature ont été testées en vue de choisir celle pouvant s'adapter le mieux à notre zone d'étude, et d'estimer par la suite, à l'aide de cette dernière et des données climatiques dont nous disposons, l'évolution de l'évapotranspiration dans le bassin versant de l'Ouergha.

Les formules qui ont fait l'objet de cette étude comparative sont celles de Thornthwaite, de Blaney-Criddle, de Turc, de Bouchet et de PriestlyTaylor. Cette étude comparative a été réalisée en utilisant les données de la région de Fès. II s'agit d'une zone où les paramètres météorologiques sont suivis de façon régulière, par la station météorologique de Fès-Saïs.

Les résultats obtenus ont conduit aux remarques suivantes :

- Les valeurs de l'évapotranspiration les plus faibles sont obtenues à partir de la formule de Thornthwaite. Une étude antérieure sur l'estimation de l'évapotranspiration, effectuée par Najjar (1982) a montré qu'en climat méditerranéen, cette formule de Thornthwaite donne des résultats sous-estimés, ce qui est conforme à nos résultats.

- La formule de Bouchet se singularise nettement par rapport aux autres: elle a tendance à surestimer fortement l'évapotranspiration. Son application s'avère délicate en raison 
de la sensibilité de Piche au vent et à son énergie advective, elle tend à surestimer l'évapotranspiration dans les zones ventilées [Bouchet, 1963].

- La formule de Priestly-Taylor, bien qu'elle donne des résultats proches de ceux de Turc et de BlaneyCriddle, semble sous-estimer l'évapotranspiration par rapport aux autres, surtout en automne et en hiver.

- La formule de Turc a l'avantage, quant à elle, de n'utiliser en tant que données nécessaires aux calculs, qu'un nombre limité de paramètres généralement disponibles (température moyenne mensuelle, insolation mensuelle et humidité relative) [Turc, 1961].

Finalement, et tenant compte des éléments précités, la formule de Turc est celle qui semble la plus adaptée à des études hydrologiques similaires à celle que nous avons mené sur le bassin de l'Ouergha. Elle est parmi les formules les plus utilisées, surtout dans l'établissement des bilans hydrologiques, et ceci en raison de sa simplicité et des bons résultats qu'elle donne au pas de temps décadaire.

Aussi, à l'aide de cette formule, une estimation de l'évapotranspiration potentielle au niveau du bassin versant de l'Ouergha, pour la période 1966-95 a été faite.

Nous avons représenté sur la figure 5, pour la période 1966-95, l'évolution interannuelle de l'évapotranspiration potentielle calculée. Ce paramètre a connu des fluctuations entre $1120 \mathrm{~mm}$ et $1260 \mathrm{~mm}$ durant cette période, ce qui correspond à une variation de $10 \%$.

Les années 1971, 1972, 1976, 1982 et 1991 ont enregistré les valeurs de l'évapotranspiration potentielle les plus faibles, alors que les

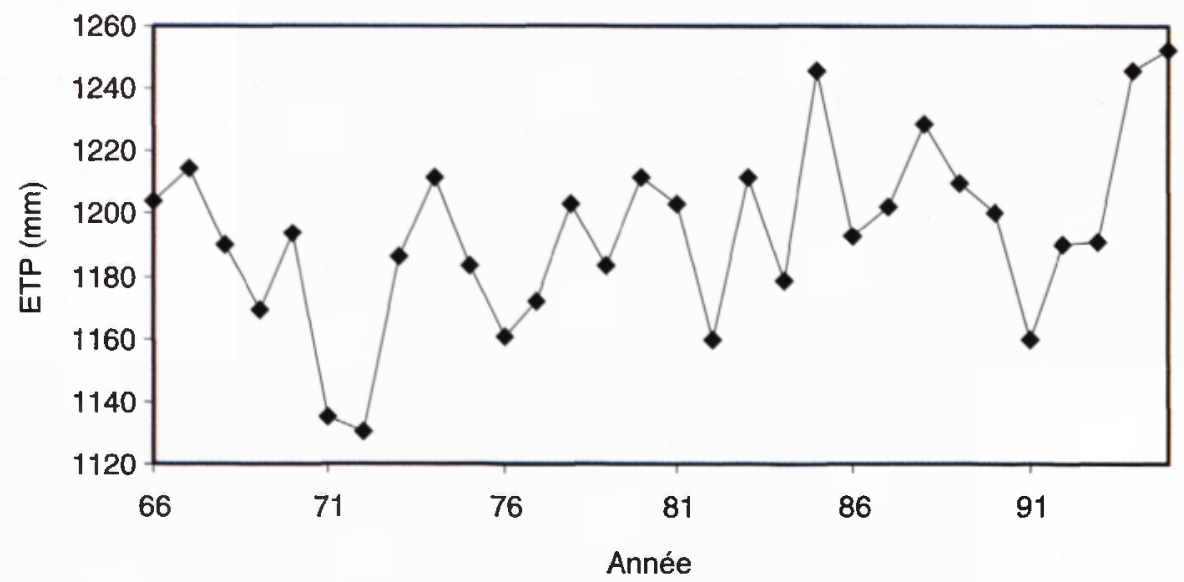

Figure 5. - Evolution interannuelle de l'évapotranspiration potentielle au niveau du bassin versant de l'Ouergha sur la période 1966-1995.

Fig. 5. - Interannual evolution of potentiel evaporation at the Ouergha watershed (1966-1995). 
années 1985, 1994 et 1995 ont enregistré quant à elles, les valeurs les plus élevées de l'évapotranspiration potentielle durant cette période.

\subsection{Données de débits}

Les données de débits dont nous avons pu disposer sont ceux relevés à l'exutoire du bassin, au niveau de la station hydromètrique de M'jara, sur la période 1934-1995.

Nous remarquons sur la figure 6 , représentant l'évolution interannuelle des débits moyens de la station M'jara, pour la période 1934-95 des fluctuations importantes du débit sur cette période (de $25 \mathrm{~m}^{3} / \mathrm{s}$ à $290 \mathrm{~m}^{3} / \mathrm{s}$ ), avec une alternance de périodes sèches et humides, dont la durée varie de 2 à 5 ans.

Nous distinguons deux types de sous-périodes : $1934-63$ et 1964-95. Durant la première sous-période, on ne remarque pas de tendance d'évolution nette du débit; par contre, audelà de 1963, et malgré la persistance de l'alternance des épisodes humides et secs, les niveaux d'eau enregistrés l'hiver ont connu une diminution permanente (ne dépassant guère les $150 \mathrm{~m}^{3} / \mathrm{s}$ au-delà de 1971), alors que ce débit fut dépassé plusieurs fois durant la souspériode 1934-63: 1941, 1942, 1947, $1951,1955,1956,1960$ et 1963 (soit plus de $25 \%$ des années).

\section{APPLICATION DU MODĖLE AU BASSIN VERSANT DE L'OUERGHA}

\subsection{Calibration}

La première étape de ce travail est la calibration du modèle. Elle a permis de déterminer les 9 paramètres d'ajustement à partir des données et

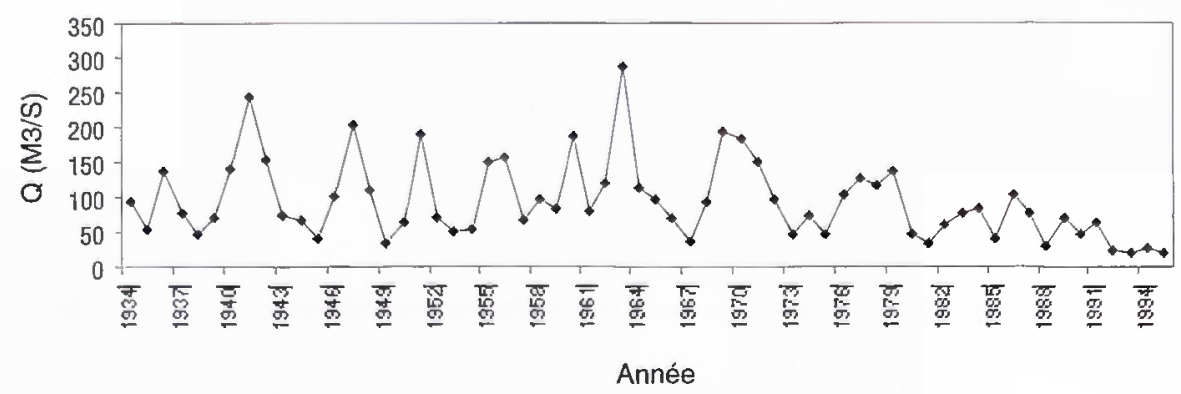

Figure 6. - Evolution interannuelle des débits à l'exutoire du bassin de l'Ouergha sur la période 1934-1995.

Fig. 6. - Interannual evolution of flows at Ouergha watershed outlet (1934-1995). 


\section{Lames mensuelles observées et calculées Calibration (Sep 66 - Août 75)}

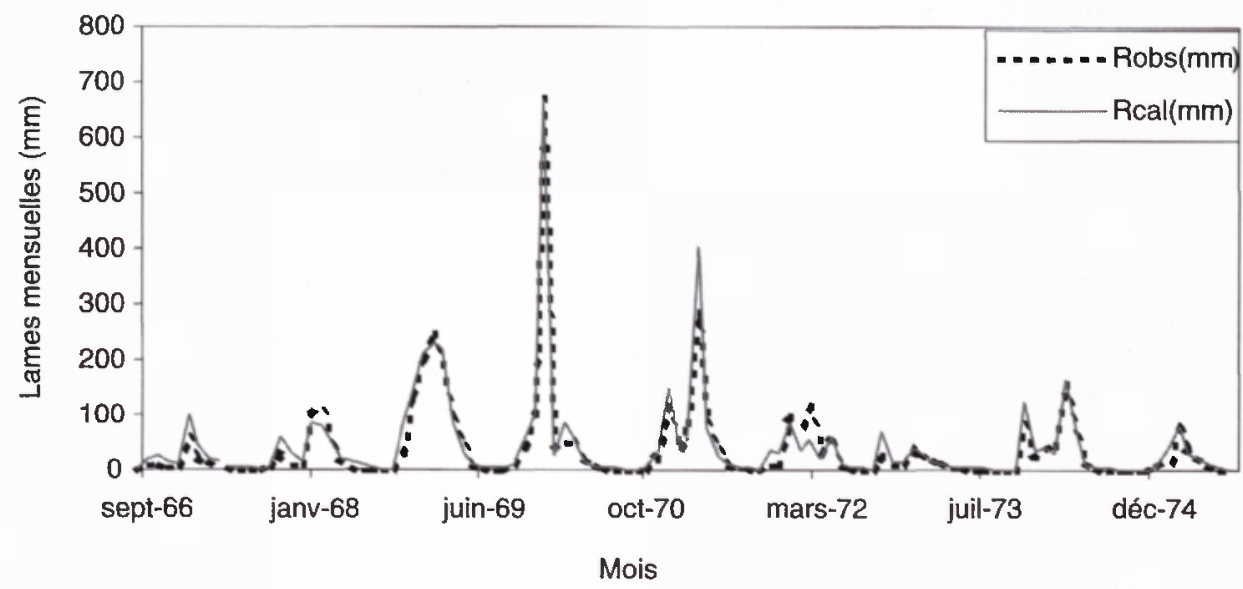

Figure 7. - Résultats du calage du modèle sur le bassin versant de l'Ouergha.

Fig. 7. - Calibration model results at Ouergha watershed.

des réponses mesurées sur le système réel.

Il s'agit d'une phase déterminante dans la modélisation. Toutefois, la qualité de la reproduction et de l'optimisation des paramètres, dépend de la fiabilité des données d'entrée observées, de la longueur et de la représentativité de la période d'observation.

La calibration est d'autant plus précise que la période d'observation est plus longue. Une période de 10 ans a été choisie (durée d'optimisation maximale acceptée par le modèle), il s'agit de la période s'étalant entre septembre 1966 et août 1975.

La figure 7 représente l'évolution des lames mensuelles observées (....) et calculées $(-)$ par le modèle pendant la période du calage. On note que le modèle retrouve les grandes tendances de l'évolution du système modélisé : II n'y a pas de sur ou sousestimation importante des lames moyennes observées.

\subsection{Validation}

Au terme de cette phase de calibration, les paramètres du modèle ainsi optimisés ont été utilisés pour la phase suivante qui est celle de la validation. La période utilisée pour cette étape du travail est celle comprise entre septembre 1975 et août 1995 (20 années).

La figure 8 représente l'évolution des lames mensuelles observées (....) et calculées (-) par le modèle pendant la période de validation. II en res- 


\section{Lames mensuelles observées et calculées Validation (Sep 75 - Août 95)}

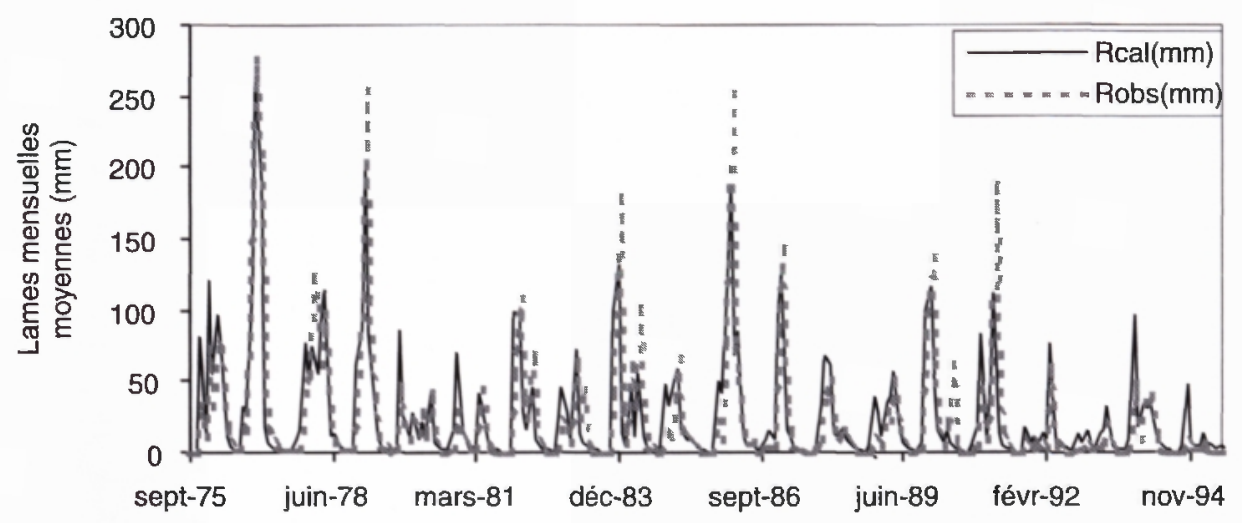

Mois

Figure 8. - Résultats de la validation du modèle sur le bassin versant de l'Ouergha.

Fig. 8. - Validation model results at Ouergha watershed.

sort une bonne concordance entre les valeurs observées et calculées, aussi bien pour les périodes d'étiage que pour les périodes de forte pluviométrie.

Pour estimer la précision des résultats du modèle élaboré, une analyse des résultats a été faite à l'échelle moyenne mensuelle, et ce, sur toute la période d'étude 1966-95.

La figure 9 représente l'évolution des lames moyennes mensuelles observées et calculées par le modèle sur toute la période d'étude 1966-95. On constate que les lames mensuelles calculées reproduisent correctement le cycle annuel observé (avec une erreur approximative de $6,4 \%$ au niveau annuel). Toutefois les mois d'octobre et de novembre enregistrent des écarts importants; ceci peut être expliqué par le phénomène de recharge de la nappe: celle-ci ayant connu un niveau de tarissement élevé durant la période estivale, les premières pluies de l'automne auront tendance à s'infiltrer plutôt qu'à ruisseler (l'humidité du sol est à son taux le plus bas).

Par ailleurs, les éléments suivants pourraient expliquer, en partie, les écarts calculs-mesures relevés sur la figure 9 :

- Le réseau pluviométrique, utilisé pour évaluer les apports journaliers en précipitations dans le bassin, ne couvre pas de façon homogène l'ensemble du bassin (figure 3) $(90 \%$ des stations utilisées sont situées dans la partie sud du bassin, caractérisé par 


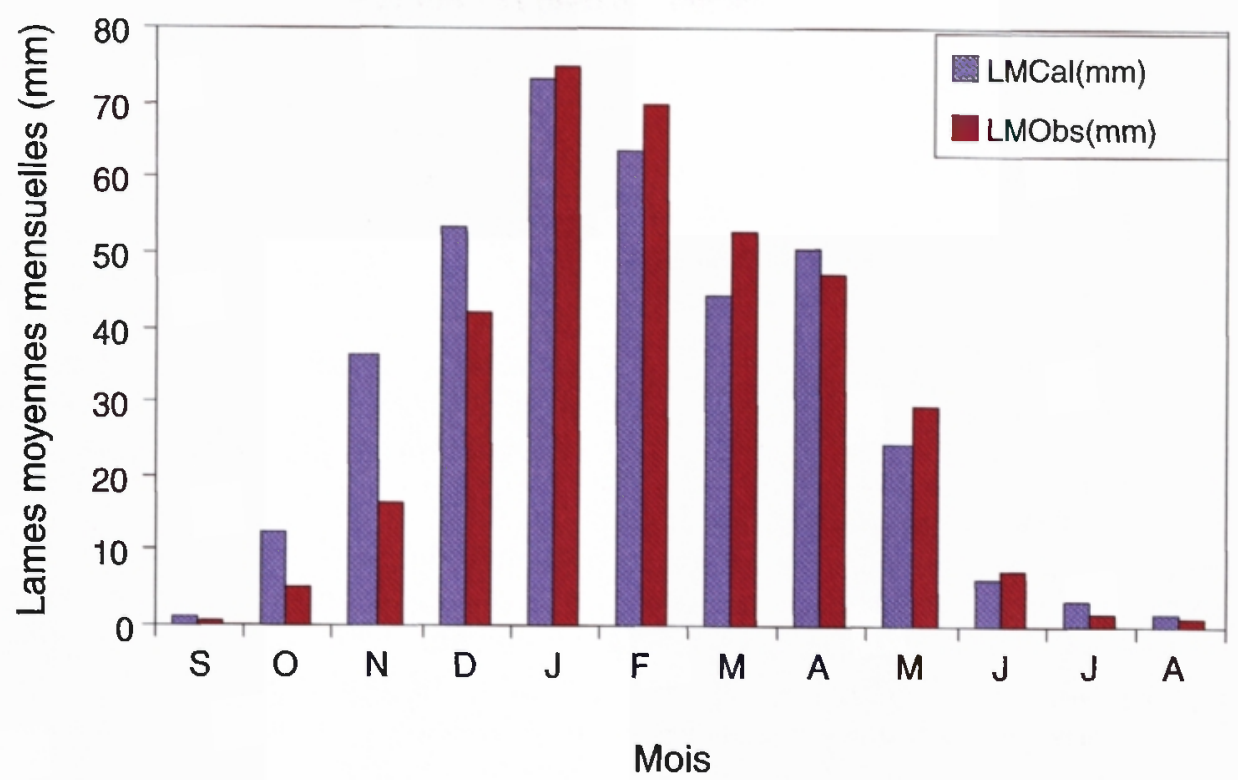

Figure 9. - Lames moyennes mensuelles observées et calculées par le modèle. Fig. 9. - Monthly mean flows observed and simulated by the model.

les plaines alors que la partie Nord est montagneuse).

- L'évaporation est considérée constante au niveau journalier : ceci n'est pas une représentation vraisemblable de la réalité, surtout pour les années sèches. En plus, son estimation a été faite à partir des températures mesurées dans une station météorologique, située à l'extérieur du bassin (station de Fès-Saïs). En effet, les températures ne sont pas mesurées de façon systématique dans les stations se situant dans le bassin.
- Le rôle et la dynamique de la nappe phréatique ne sont pas bien connus pour ce bassin.

\section{COMPORTEMENT DU BASSIN DE L'OUERGHA EN CAS DE CHANGEMENT CLIMATIQUE}

Malgré les incertitudes qui persistent encore à l'heure actuelle en ce qui concerne l'ampleur et les délais exacts des changements climatiques; il est dés à présent nécessaire d'évaluer comment le cycle hydrologique pourrait évoluer, afin de prévoir des 
stratégies d'adaptation. Ceci est particulièrement important pour un pays comme le Maroc où la ressource eau est rare.

Le Maroc dispose d'un climat aride à semi-aride, où les températures de l'air dépassent $40^{\circ} \mathrm{C}$ dans certaines régions pendant l'été, ce qui donne un poids non négligeable à l'évapotranspiration et à son influence sur le bilan hydrique des bassins versants de la région.

Ces températures pourraient connaître des niveaux plus importants selon les résultats les plus récents des Modèles de Circulation Générale [IPCC, 1995], [IPCC, 1997], en cas de confirmation du réchauffement planétaire, avec ce que ceci pourrait engendrer comme impact sur les ressources hydriques nationales.

Nous avons cherché à évaluer les incidences possibles du réchauffement du Maroc en cas de dédoublement de la concentration atmosphérique du $\mathrm{CO}_{2}$ sur le potentiel en eaux douces, plus particulièrement au niveau du barrage Al Wahda, qui représente à lui seul, plus de $30 \%$ des eaux de surfaces mobilisées : il s'agit d'une infrastructure d'une grande importance tant pour la production hydroélectrique que pour l'eau potable et l'irrigation agricole.

\section{Scénarios envisagés selon les ré- sultats des MCG}

Les Modèles de Circulation Générale (MCG), simulant l'évolution à long terme du climat planétaire, prévoient pour la région du Maghreb, pour une concentration de $\mathrm{CO}_{2}$ double dans l'atmosphère $\left(2^{*} \mathrm{CO}_{2}\right)$, une augmentation de la température de l'air allant de 1 à $2^{\circ} \mathrm{C}$ en été, et de 2 à $3^{\circ} \mathrm{C}$ en période hivernale. Les prévisions de ces modèles quant à la variation du niveau des précipitations dans la région du Maghreb restent à ce jour incertaines. Aussi, nous n'avons considéré dans cette étude aucun changement de précipitations.

A partir de l'ensemble de ces éléments, nous avons considéré 2 scénarios du comportement à long terme(vers 2050) de la température dans notre région que nous avons testé à l'aide du modèle hydrologique présenté ci-dessus, et ce, pour le bassin versant de l'Ouergha.

Les scénarios adoptés consistent à considérer les données climatiques de la période 1966-1995, auxquelles nous avons porté les modifications suivantes :

Scénario $1: \Delta \mathrm{T}=1^{\circ} \mathrm{C}$ (en juin, juillet et août).

janvier et février).

$\Delta \mathrm{T}=2^{\circ} \mathrm{C}$ (en décembre,

Pour les autres mois de l'année, le réchauffement a été obtenu par interpolation linéaire à partir des données précédentes.

Scénario 2: $\Delta \mathrm{T}=2^{\circ} \mathrm{C}$ (en juin, juillet et août). janvier et février). 


\section{Scénario 1(1966 1995)}

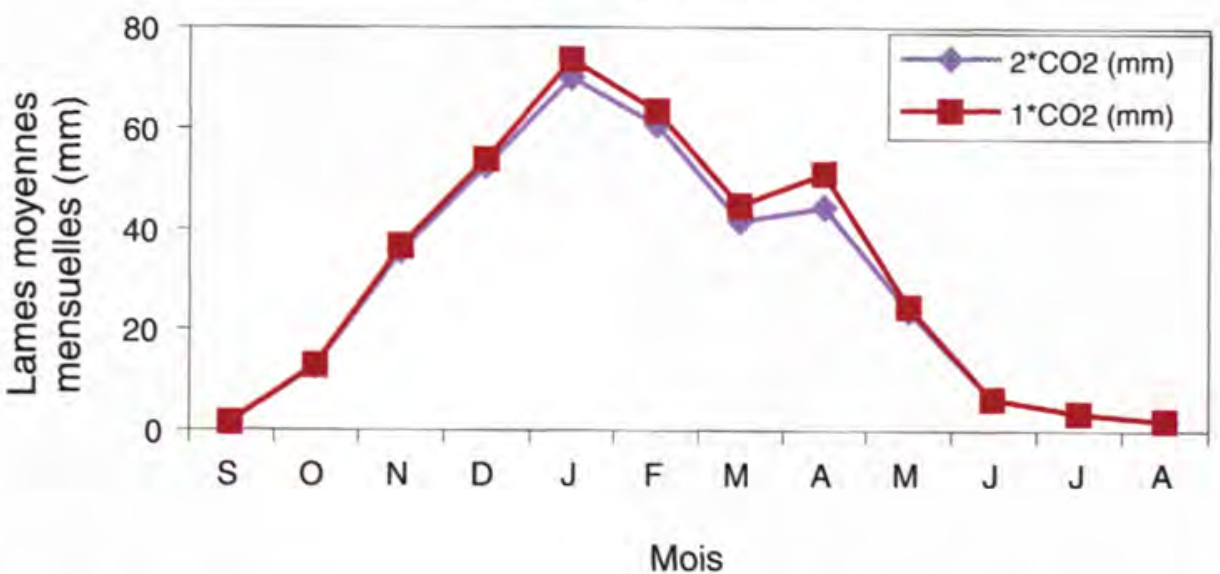

Figure 10. - Lames moyennes mensuelles du bassin versant de l'Ouergha calculées $\left(1^{*} \mathrm{CO}_{2}\right)$ et simulées par le modèle en cas d'un échauffement de 1 à $2^{\circ} \mathrm{C}$ (1966-1995).

Fig. 10. - Monthly mean flows of Ouergha watershed calculated $\left(1^{\circ} \mathrm{CO}_{2}\right)$ and simulated by the model in the case of warming from 1 to $2^{\circ} \mathrm{C}(1966-1995)$.

\section{Scénario 2}

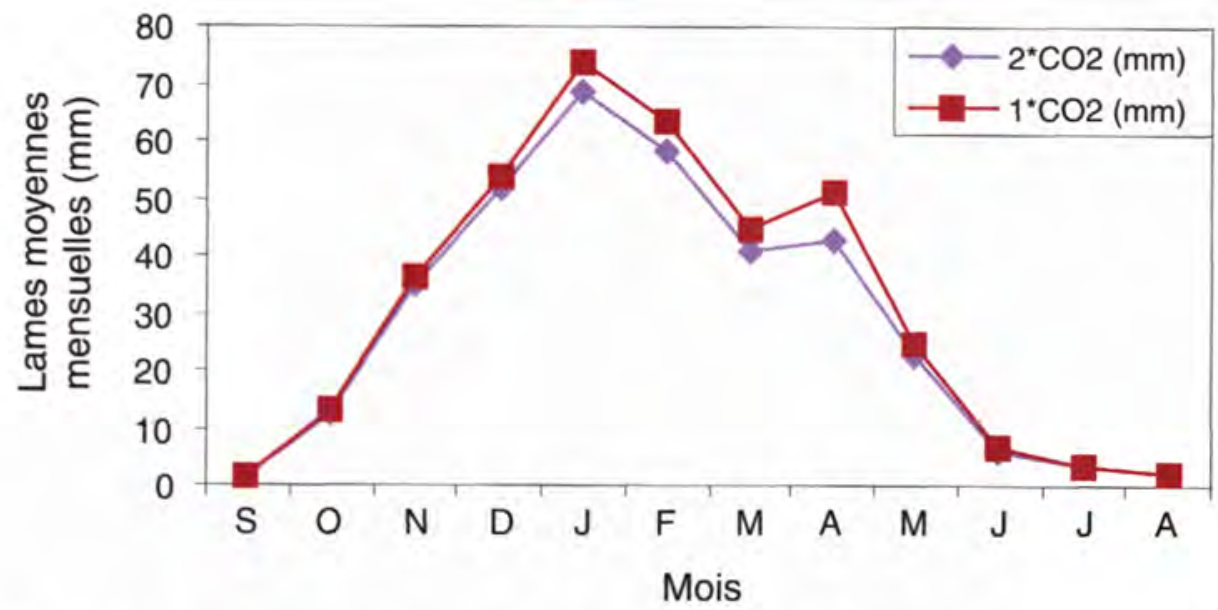

Figure 11. - Lames moyennes mensuelles du bassin versant de l'Ouergha calculées $\left(1^{*} \mathrm{CO}_{2}\right)$ et simulées par le modèle en cas d'un échauffement de 2 à $3^{\circ} \mathrm{C}$ (1966-1995).

Fig. 11. - Monthly mean flows of Ouergha watershed calculated $\left(1^{*} \mathrm{CO}_{2}\right)$ and simulated by the model in the case of warming from 2 to $3^{\circ} \mathrm{C}(1966-1995)$. 
De même, une interpolation linéaire a été effectuée pour les autres mois de l'année.

Les lames moyennes mensuelles calculées pour les conditions actuelles et simulées avec les données météorologiques estimées pour une hypothèse d'un échauffement de 1 à $2^{\circ} \mathrm{C}$ sont présentées sur la figure 10 .

On observe sur cette figure un déficit dans le bilan d'eau, qui se distingue plus en hiver et printemps qu'en période estivale où les changements ne sont pas très notables. La diminution de la lame d'eau moyenne mensuelle est particulièrement nette pendant le mois d'avril, où elle atteint une valeur de l'ordre de $12,7 \%$.

De même, nous avons tracé sur la figure 11 , l'évolution saisonnière de la lame mensuelle moyenne pour les conditions $\left(1^{*} \mathrm{CO}_{2}\right)$ et pour le scénario 2 (échauffement de 2 à $3^{\circ} \mathrm{C}$ ).

L'écart entre les valeurs des lames mensuelles calculées sous l'hypothèse $\left(1^{*} \mathrm{CO}_{2}\right)$ et les lames mensuelles moyennes simulées pour le scénario 2 atteint les 16,6\%.

Notons que pour les deux scénarios 1 et 2 , le calcul effectué dans l'hypothèse du réchauffement prévoit des lames d'eau à tout moment inférieures à celles relatives aux conditions actuelles. Ceci est logique étant donné que le réchauffement induirait une évapotranspiration plus importante, et par la suite un bilan d'eau déficitaire.

Notons par ailleurs une réponse hydrique différentielle du bassin versant aux changements thermiques envisagés entre l'hiver - printemps et l'été - automne. Ceci peut être attribué :

- Aux scénarios considérés qui prévoient un réchauffement plus important en hiver - printemps qu'en été, d'où un impact certainement plus fort sur l'évapotranspiration.

- Aux faibles valeurs des réserves hydriques disponibles pour l'évaporation en période estivale, retardant ainsi le remplissage de la nappe à l'automne.

\section{IMPACT POSSIBLE DE CES SCÉNARIOS SUR LES RÉSERVES EN EAU DU BARRAGE AL WAHDA}

Pour mieux cerner cet impact possible d'un dédoublement de $\mathrm{CO}_{2}$ atmosphérique sur le barrage $\mathrm{Al}$ Wahda, ces 2 scénarios ont été appliqués afin de mesurer leurs incidences sur les réserves annuelles en eau reçues par le barrage.

Sur la figure 12, nous avons tracé les écarts des volumes d'eau emmagasinés, en moyenne annuelle, à Al Wahda à l'état actuel $\left(1^{*} \mathrm{CO}_{2}\right)$ et lors de l'état perturbé (échauffement) pour les deux scénarios. Nous remarquons sur cette figure que ces écarts sont plus importants lors des périodes d'hiver et de printemps, par contre, lors de la période estivale, cet écart devient moins important.

Les écarts les plus élevés ont été enregistrés pendant le mois d'avril, où une différence des volumes stockés dans le barrage dans les conditions 


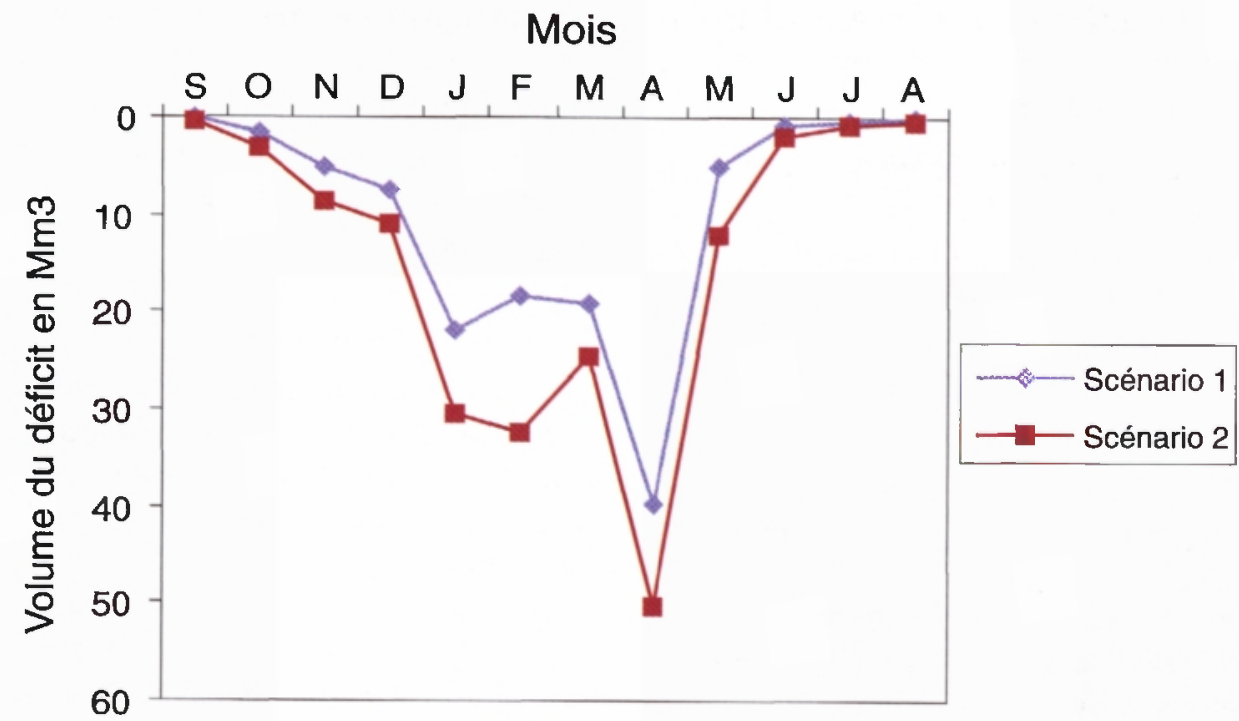

Figure 12. - Ecart des apports mensuels au barrage $\mathrm{Al}$ Wahda entre l'état $\left(1{ }^{*} \mathrm{CO}_{2}\right)$ et dans le cas d'un échauffement de 1 à $2^{\circ} \mathrm{C}$ (scénario 1) et d'un échauffement de 2 à $3^{\circ} \mathrm{C}$ (scénario 2). Fig. 12. - Difference between the monthly inflows of $\left(1{ }^{*} \mathrm{CO}_{2}\right)$ state and in the case of warming from 1 to $2^{\circ} \mathrm{C}$ (scenario 1) and in the case of warming from 2 to $3^{\circ} \mathrm{C}$ (scenario 2) to Al Wahda dam.

actuelles, et dans le cas du scénario 2 atteint $50 \mathrm{Mm}^{3}$.

Sur le tableau I suivant, nous retrouvons les apports annuels au barrage dans les conditions climatiques actuelles, et dans le cas d'un dédoublement de $\mathrm{CO}_{2}$ pour les 2 scénarios.

Le barrage Al Wahda reçoit pour une année moyenne, un apport de 2300 millions de $\mathrm{m}^{3}$ calculée sur la période 1966-95. Cette capacité connaîtrait un déficit d'environ $5 \%$ dans le cas du premier scénario. Si le deuxième scénario se réalise, ce volume reçu annuellement par le barrage baisserait jusqu'à atteindre une valeur de 2123 millions de $\mathrm{m}^{3}$, soit un déficit approximativement de $8 \%$.

\section{CONCLUSION ET PERSPECTIVES}

L'augmentation constante de la concentration des gaz à effet de serre pourrait entraîner, dans le futur, un changement progressif du climat. Des impacts non négligeables sur le cycle de l'eau devraient également accompagner ces changements climatiques.

Cette tentative de modélisation de l'interaction à l'échelle mensuelle entre paramètres climatiques (précipita- 
Tableau 1. - Apports annuels au barrage Al Wahda - Etat actuel et cas d'un échauffement de 1 à $2{ }^{\circ} \mathrm{C}$ (scénario 1) et d'un échauffement de 2 à $3^{\circ} \mathrm{C}$ (scénario 2).

Table 1. - Annual inflows to Al Wahda dam: Actual state and in case of warming from 1 to $2{ }^{\circ} \mathrm{C}$ (scenario 1) and in case of warming from 2 to $3^{\circ} \mathrm{C}$ (scenario 2)

\begin{tabular}{l|c|c|c|c}
\hline \multicolumn{1}{c|}{} & \multicolumn{2}{|c|}{ BARRAGE AL WAHDA } & \multicolumn{2}{c}{} \\
\cline { 2 - 4 } & Etat $1 \mathrm{CO}_{2}$ & Etat $2 \mathrm{CO}_{2}$ & \\
\hline Année moyenne & Volume $\left(10^{6} \mathrm{~m}^{3}\right)$ & Volume $\left(10^{6} \mathrm{~m}^{3}\right)$ & $\begin{array}{c}\text { Déficit entre } \\
1 \text { et 2 }\left(10^{6} \mathrm{~m}^{3}\right)\end{array}$ & Déficit en (\%) \\
\hline Scénario 1 & 2300 & 2179 & 121 & 5,24 \\
\hline Scénario 2 & 2300 & 2123 & 177 & 7,70 \\
\hline
\end{tabular}

tions, températures) et paramètres hydrologiques (ruissellement) au niveau d'un bassin versant a globalement permis d'obtenir un outil acceptable pour toutes prévisions futures de cette synergie.

Les résultats obtenus auraient pu être plus précis, comme cela avait été obtenu dans d'autres recherches menées ailleurs [Morin et al., 1992], [Ottlé, 1998] si :

- Les températures étaient mesurées de façon systématique dans toutes les stations hydrologiques.

- Le réseau pluviométrique couvrait mieux d'une façon homogène le bassin $(90 \%$ des stations utilisées sont situées dans la partie sud du bassin) (figure 3 ).

Les simulations réalisées dans une seconde phase de cette recherche, en vue de tester la sensibilité du potentiel hydrique de ce bassin aux fluctuations climatiques, et en particulier aux changements globaux pouvant survenir en cas de réchauffement sont évidemment à prendre avec prudence : les incertitudes qui persistent sur le réchauffement envisagé à l'échelle régionale restent à ce jour importantes.

Toutefois, même avec les hypothèses les plus optimistes, les calculs réalisés donnent un déficit hydrique, que pourrait engendrer le réchauffement, de l'ordre de $8 \%$, ce qui n'est pas négligeable pour un pays qui, même sans ces problèmes de réchauffement climatique, est voué à une pénurie hydrique à l'horizon 2025 [Mokssit et al., 1998]. Notons que les calculs similaires réalisés pour d'autres fleuves dans le monde indiquent une diminution des débits pour la plupart, supérieure à cette estimation : $11 \%$ pour le $\mathrm{Nil}, 31 \%$ pour la rivière du Niger et $43 \%$ pour la rivière Indus au Pakistan [IPCC, 95].

Une amélioration de la précision des résultats peut être réalisée si d'une part, nous disposons d'une base de données plus complète et plus homogène, et d'autre part, si les 
scénarios effectués par les modèles de circulation générale étaient plus précis à l'échelle régionale.

Après cette première tentative, pour un bassin dit humide, une étude similaire pourrait être menée sur un bassin versant semi-aride ou aride afin de voir qu'elle pourrait être la réponse de ces bassins devant ces mêmes changements climatiques, et par la suite essayer d'extrapoler les résultats trouvés à tous les types de bassins. On aura ainsi, un aperçu du devenir des ressources en eau du Maroc, en réponse aux changements climatiques.

\section{RÉFÉRENCES BIBLIOGRAPHIQUES}

Agoumi A. (1995). "Variabilité climatique et ressources en eau au Maroc", communication présentée lors du colloque international «Eau: Gestion de la rareté ", organisé par l'Amicale des Ingénieurs Marocains des Ponts et Chaussées, les 19 et 20 octobre 1995.

Agoumi A. (1998). "Changements climatiques et ressources en eau dans les pays du Maghreb". Projet RAB/G31 PNUD/FEM.

Agoumi A., Yacoubi M., Senoussi S., Chikri N., Mokssit M. (1997). "Comportement hydro-climatique du bassin versant de l'Ouergha: Estimation de l'évapotranspiration». Revue Marocaine de Génie Civil, N ${ }^{\circ} 72$, décembre 1997.

Bouchet R.J. (1963). «Evapotranspiration réelle et potentielle: signification climatique ». Proc. Gen. Assembly IAHS, Berkeley, Publ 62, p. 134-142.

DGH (1995). «Etude hydrologique de prévision des crues au site du barrage Al Wahda". Rapport établi par la Di- rection Générale de l'Hydraulique Maroc.

H.O.M. (1989). Hyrrom Operation Manual - Institute of Hydrology, Wallingford United Kingdom.

IPCC (Intergouvernemental Panel of Climate Change), premier rapport d'évaluation, Vol. I - août 1995.

IPCC (1997). "The regional impact of climate change : An assessement of vulnerability». Rapport du groupe de travail II.

Karas J. (1997). «Le changement climatique et le bassin méditerranéen ». Rapport préparé pour Greenpeace.

Mandelkern S. (1994). "Les conséquences hydrologiques de l'effet de serre ". Rapport EDF, HE - 43/94/019/A.

Mokssit A., Benbiba A., Ouldbba A., Benabdelfadel A., Bensaid F. (1998). "Changements climatiques et ressources en eau au Maroc». Rapport établi dans le cadre du projet PNUDFEM RAB /94/G31.1998.

Morin G., Slivitsky M. (1992). «Impacts de changements climatiques sur le régime hydrologique : le cas de la rivière Moisie ». Revue Internationale des Sciences de l'Eau, Vol. 5, № 2.

Najjar G. (1982). « Méthode de cartographie de l'évapotranspiration journalière en moyenne montagne tempérée : Application au bassin versant de Ringelback (Hautes Vosges)". Thèse de $3^{\text {e }}$ cycle, Université Louis Pasteur - Strasbourg.

Nascimento N.O. (1995). «Appréciation à l'aide d'un modèle empirique des effets d'actions anthropiques sur la relation pluie-débit à l'échelle d'un bassin versant ». Thèse soutenue en juillet 1995 à l'Ecole Nationale des Ponts et Chaussées.

Ottlé C. (1998). «Le programme GewexRhône: Modélisation couplée (atmosphère - surface) de l'écoulement". Lettre Pigb - Pmrc - France, janvier 1998. 
Perennes J.J. (1993). «L'eau et les hommes au Maghreb» - Collection "Hommes et Sociétés" - Editions Karthala.
Turc L. (1961). «Evaluation des besoins en eau d'irrigation, évapotranspiration potentielle». Ann. Agro, 12(1), p. 1349.

\author{
LOUIS - JEAN \\ avenue d'Embrun, 05003 GAP cedex \\ Tél. : 04.92.53.17.00 \\ Dépôt légal : 403 - Juin 2000 \\ Imprimé en France
}

